\title{
Numerical Simulation of Free Convection Heat Transfer of Ferrofluid in an Oval Shaped Closed Loop
}

\author{
Jaswinder Singh Mehta ${ }^{1}$, Rajesh Kumar ${ }^{2}$, Harmesh Kumar ${ }^{3}$ and Harry Garg ${ }^{4}$ \\ ${ }^{1,2 \& 3}$ Department of Mechanical Engineering, UIET, PU, Chandigarh, India \\ ${ }^{4}$ CSIR-CSIO, Sector 30, Chandigarh, India \\ E-Mail: jsmehta@pu.ac.in
}

\begin{abstract}
Free convective heat transfer capability of kerosene based ferrofluid flowing through an oval shaped twodimensional closed loop has been investigated numerically. COMSOL Multi Physics, a standard CFD code has been applied for solving the governing equations. A constant magnetic field was applied using permanent magnet and time dependent numerical study has been conducted for laminar fluid flow and heat transfer. The fluid was found to flow under the effect of externally applied magnetic field and spatially varying temperature. Maximum velocity of $4.43 \mathrm{~mm} / \mathrm{s}$ has been found under the influence of externally applied magnetic field generated by the permanent magnet and flow was observed to be continuous. Temperature and velocity plots have also been plotted reconfirming the candidature of ferrofluid as a coolant for heat transfer applications of mini/micro devices.
\end{abstract}

Keywords: Ferrofluid, Convective Heat Transfer, Thermomagnetic Convection, Kelvin body Force

\section{INTRODUCTION}

Last few decades have witnessed unprecedented growth in the computational power of electronic devices, thereby generating high temperatures during their working. Conventional cooling methods prove to be inadequate in handling high heat flux generated by these devices, thus necessitating the need for smart cooling devices, which can remove heat at such a rate that they can work without breaking down. Ferrofluid driven heat exchangers can provide a potential solution for cooling of such miniaturized devices.

Ferrofluid is colloidal mixture of nano-sized magnetic particle coated with surfactant in a non-magnetic base fluid. The particles are coated with absorbed surfactant layers so as to keep a stable suspension state and to avoid agglomeration.

Ferrofluid consist of single domain magnetic nanoparticles which are randomly oriented in the carrier fluid. Thus, when such a fluid is subjected to thermal gradient in the presence of an external magnetic field, due to non-equilibrium magnetization in the fluid, the fluid will experience a magnetic body force, called "Kelvin body force" which results in flow of fluid in the direction of higher temperature section [1].

Shakiba and Vahedi [2] studied the effect of non-uniform magnetic field on hydrothermal characteristics of water- based ferrofluid flowing through a horizontal double-pipe heat exchanger using ANSYS Fluent. Increase in Nusselt number was observed with increase in the intensity of magnetic field.

Aminfar et al. [3] numerically examined the hydrothermal behaviour of water-based ferrofluid flowing through a helical channel using the $\mathrm{CV}$ technique. Transverse magnetic field was applied to the channel and increase in the flow rate and velocity gradient was observed with augmentation in heat transfer.

Goharkhah et al. [4] performed numerical simulations using COMSOL to find efficient arrangement and locations of four electromagnets along the channel for water based ferrofluid flowing through a channel with evenly heated upper and lower $\mathrm{Cu}$ parallel plates. The average heat transfer coefficient was found to augment with the frequency of magnetic field and Re.

Wrobel et al. [5] studied numerically and experimentally thermo-magnetic convective flow behaviour of paramagnetic fluid in an annular enclosure with a round rod core and a cylindrical outer wall. Results show that magnetizing force affects the heat transfer rate and magnetic convection of paramagnetic fluid can be controlled by a strong magnetic field.

R. Zanella et al. [6] numerically studied the transformer cooling using ferrofluid.Vegetable oil seeded with magnetite nanoparticles @ 10\% volume fraction was considered. A magnetohydrodynamics code called SFEMaNS was applied on a solenoid system and magnetostatic, Navier-Stokes, and energy equations were solved simultaneously. Numerical results for ferrofluid point out that the magnetoconvection modifies the flow convection pattern and speed and subsequenlty temperature rise in the coil was reduced by about $9.4 \%$ with ferrofluid cooling.

E. Aursand et al.[7] proposed a one-dimensional multiphase flow model for thermomagnetically pumped ferrofluid that includes the effects of heat transfer, friction, gravity, and magnetic forces. The proposed model was also validated against experiments from the literature and was found to give good predictions for the thermomagnetic pumping performance. However, the results also signify 
very large sensitivity to uncertainties in heat transfer coefficient predictions.

Tomasz Strek [8] considered the 2D, laminar and incompressible ferrofluid flow between two parallel flat plates. Flow was influenced by magnetic dipole sufficient enough to saturate the ferrofluid using computational fluid dynamics code COMSOL. Heat source in the form of rectangular blocks was placed below the upper wall. It was observed that under the action of Kelvin Body force generated due to interaction between the local magnetic field and the molecular magnetic moments, hotter fluid is being displaced by colder fluid when the magnet is placed near to heat source, thus system as a whole does not require additional energy input except energy from external magnet. Wahid Cheriefet al. [9] experimentally analyzed the effect of parameterssuch as the direction of magnetic field and its level, length of the magnetic source, particle concentration and the mass flow rate on convective heat transfer coefficient and pressure drop for a ferrofluid flowing through a square duct under magnetic field.Augmentation in value of $\mathrm{h}$ and pressure drop was observed with increase in volume fraction, and low mass flow rates were considered more effective as far as cooling capability is concerned. Magnetic source length has lesser effect on pressure drop; however, heat transfer coefficient is strongly influenced by its length. In addition, best results for convective heat transfer coefficients were achieved when magnetic field washeld perpendicular to the heat flux.

The work presented in this paper explores the possibility of ferrofluid to be used as a coolant for heat dissipation of miniaturized devices. Numerical simulation is carried out using COMSOL MultiPhysics 5.0. Governing equations such as momentum and energy equations have been accordingly modified by incorporating term containing magnetic force. Simulations are performed on 2D oval shaped closed loop and results are plotted in the form of velocity and temperature plots.

\section{NUMERICAL MODELLING}

\section{A. Description of Geometry}

A two dimensional model as shown in Fig. 1 is used for simulation study. The model consists of a oval shaped closed loop through which kerosene based ferrofluid would flow, a permanent magnet for applying magnetic field, accumulator acting as a reservoir for the fluid, a heat source and fins for dissipating heat to the surroundings.

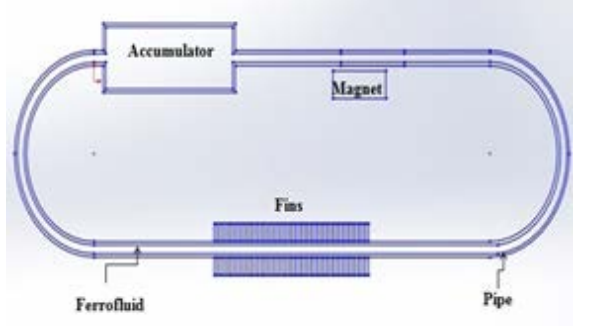

Fig.1 Two dimensional geometrical model

\section{B. Equations governing ferrofluid}

Following equations govern the flow of ferrolfuid for the problem considered for analysis.

Continuity Equation:

$$
\frac{\partial \rho}{\partial \mathrm{t}}+\nabla \cdot(\rho \mathrm{u})=0
$$

Momentum Equation:

$$
\begin{aligned}
& \rho \partial \mathrm{u} / \partial \mathrm{t}+\rho \mathrm{u} . \nabla \mathrm{u}=-\nabla \mathrm{p}+\nabla \cdot\left(\mu\left(\nabla \mathrm{u}+(\nabla \mathrm{u})^{\wedge} \mathrm{T}\right)-\right. \\
& 2 / 3 \mu(\nabla \cdot \mathrm{u}) \mathrm{I}+\mathrm{F}
\end{aligned}
$$

Energy equation:

$$
\rho c_{p}\left(\frac{\partial T}{\partial t}+u . \nabla \mathrm{T}\right)=k \nabla^{2} T+\mu \Phi+\dot{q}(1.3)
$$

Magnetic Induction:

$$
B=\mu_{\circ}(H+M)(1.4)
$$

Kelvin Body Force:

$$
\mathrm{F}=(\mathrm{M} . \nabla) \mathrm{B}
$$

The properties of ferrofluid used for the simulation is presented in Table I.

TABLE I FERROFLUID PROPERTIES

\begin{tabular}{|c|l|l|}
\hline $\begin{array}{r}\text { Sr. } \\
\text { No }\end{array}$ & \multicolumn{1}{|c|}{ Parameters } & \multicolumn{1}{|c|}{ Value } \\
\hline 1 & Viscosity, $\mu$ & $0.001 \mathrm{~N}-\mathrm{s} / \mathrm{m}^{2}$ \\
\hline 2 & Density, $\rho$ & $909.9 \mathrm{~kg} / \mathrm{m}^{3}$ \\
\hline 3 & Thermal conductivity, $\mathrm{k}$ & $0.17 \mathrm{~W} /(\mathrm{m}-\mathrm{K})$ \\
\hline 4 & Remanant flux density & $1.00 \mathrm{~T}$ \\
\hline 5 & Relative permeability of magnet & 5000.0 \\
\hline 6 & Surrounding temperature & $293.15 \mathrm{~K}$ \\
\hline 7 & Curie temperature & $350 \mathrm{~K}$ \\
\hline 8 & Magnetic susceptibility & 0.3860 \\
\hline 9 & $\begin{array}{l}\text { Relative permeability of } \\
\text { fluid }\end{array}$ & 1.3860 \\
\hline
\end{tabular}

\section{Simulation}

Geometry is created in model builder of COMSOL Multiphysics 5.0 and materials/material properties are assigned to different domains inputting initial values followed by mesh generation. Physics controlled finer mesh with following statistics is created.

TABLE II Meshing Statistics 


\begin{tabular}{|l|l|l|}
\hline $\begin{array}{c}\text { Sr. } \\
\text { No. }\end{array}$ & \multicolumn{1}{|c|}{ Property } & \multicolumn{1}{|c|}{ Value } \\
\hline 1 & Minimum element quality & 0.1173 \\
\hline 2 & Number of elements & 23325 \\
\hline 3 & Number of vertex elements & 352 \\
\hline 4 & Number of boundary elements & 3480 \\
\hline
\end{tabular}

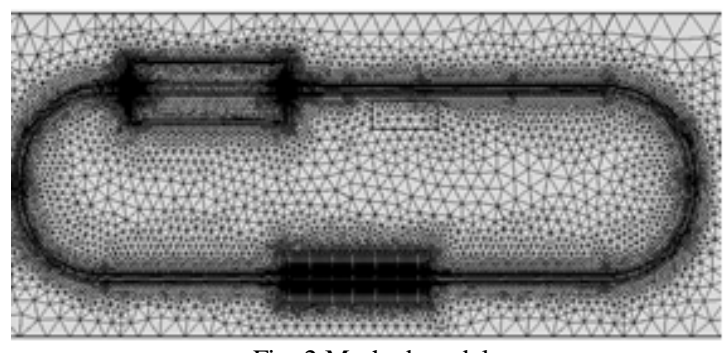

Fig. 2 Meshed model
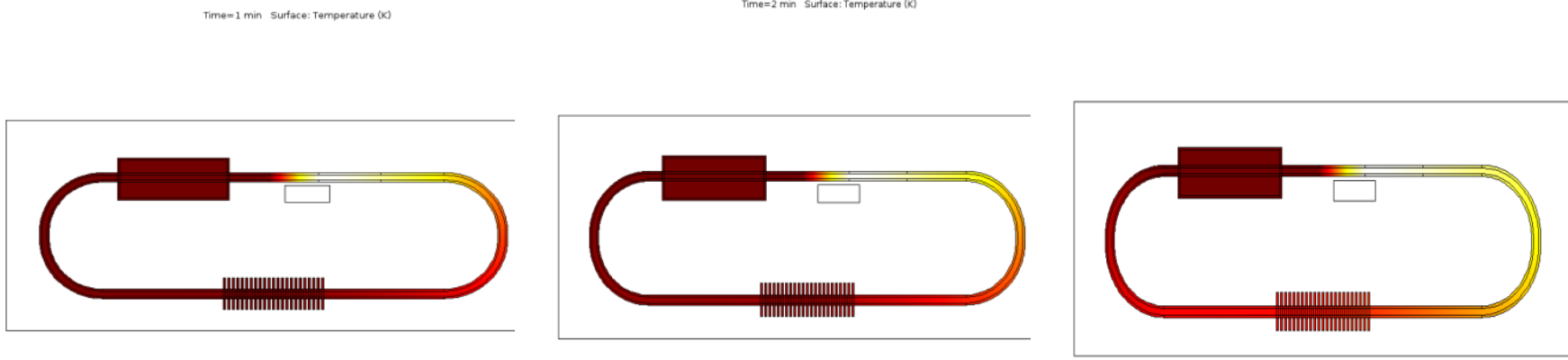

(a)

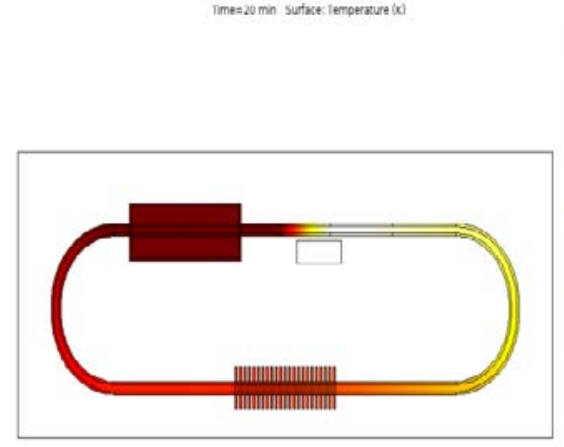

(d) (b)
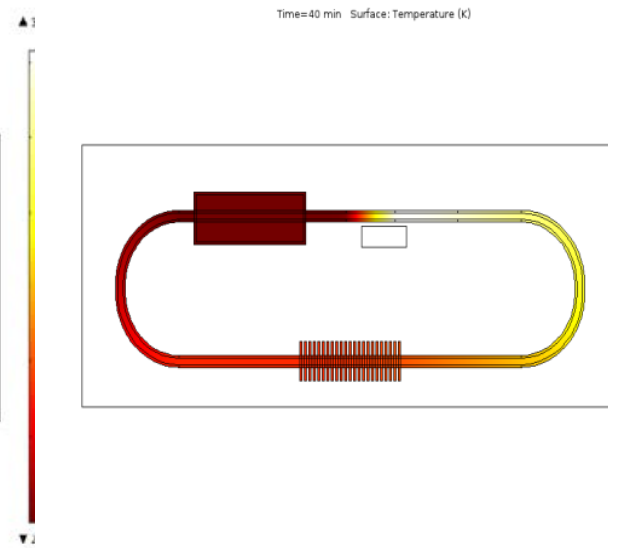

(e)
Since the model involves different physics like magnetic field, heat transfer in solids and laminar flow, thus different physics are combined using conjugate heat transfer option available in COMSOL. Conjugate heat transfer corresponds with the combination of heat transfer in solids and heat transfer in fluids. Magnetic field is considered to be generated by permanent magnets of intensity one Tesla and treated stationary, while heat transfer and laminar flow are treated as time dependent while solving the model.

\section{RESULTS AND DISCUSSIONS}

Simulation results of temperature generated and velocity produced at different length of time are presented in Fig. 3 and Fig. 4 respectively. . 


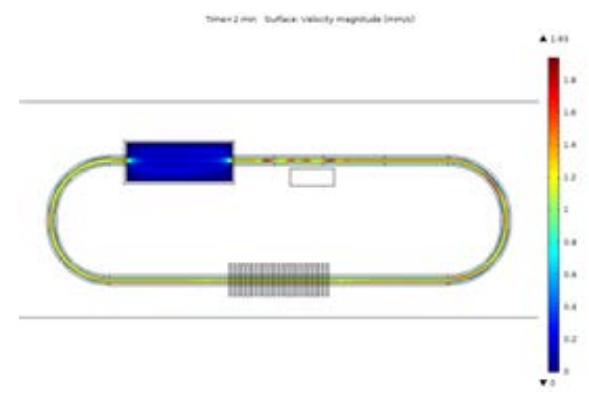

(a)

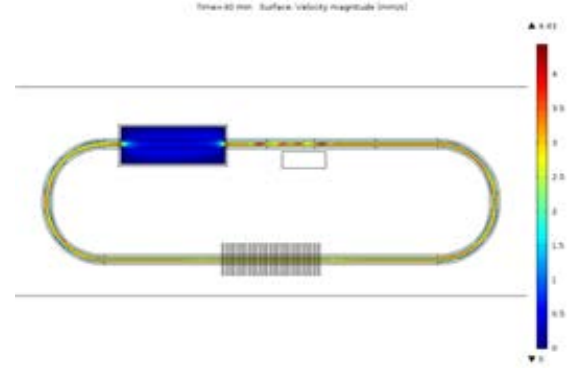

(d)

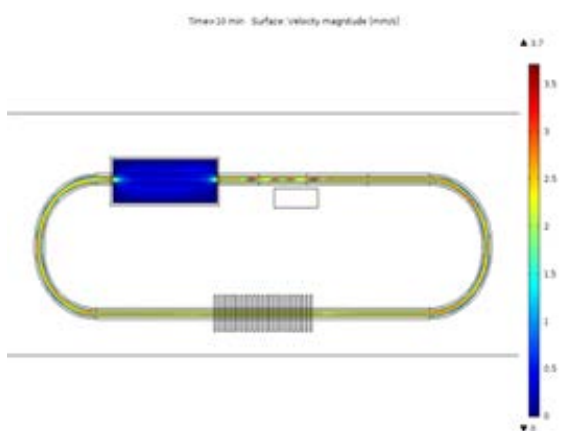

(b)

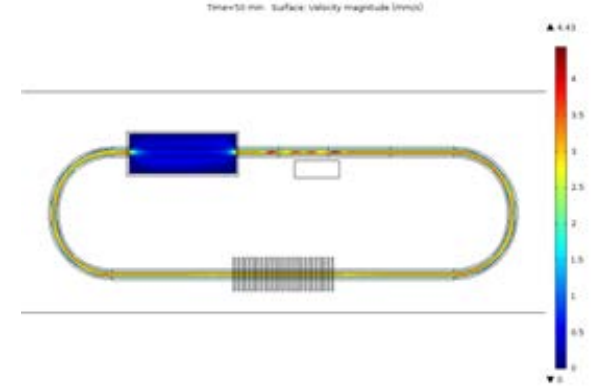

(e)

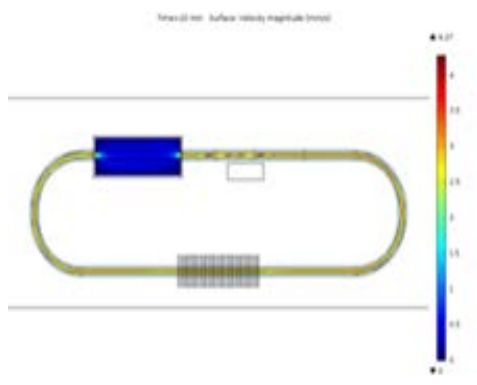

(c)

Fig. 4. Fluid Velocity contours (mm/s) generated at different point of time (a) $2 \mathrm{~min}$ (b) $10 \min$ (c) $20 \mathrm{~min}$ (d) $40 \mathrm{~min}$ (e) $50 \mathrm{~min}$.

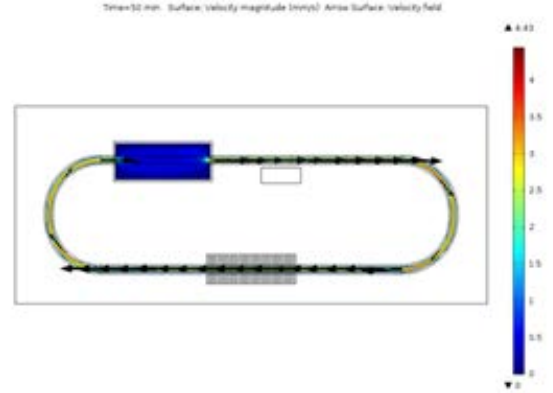

Fig. 5. Velocity vectors representing direction of fluid flow in the loop

\section{CONCLUSION}

From the numerical study, following conclusions could be drawn:

1. Temperature plots showing variations of temperature in different parts of loop confirm the removal of heat flux by the ferrofluid, establishing flow of the fluid. Cold fluid is available for extracting heat from the heat source every time after one loop cycle as represented in Fig. 3.

2. Steady state is established after $t=40$ minutes, as there are minimal or no variations in the temperature profile after that as depicted in Fig 3(e) and Fig. 3(f).

3. Maximum velocity of $4.43 \mathrm{~mm} / \mathrm{s}$ is found at $\mathrm{t}=40$ minutes i.e. fluid moves under the influence of external magnetic field generated by the permanent magnet of intensity $1 \mathrm{~T}$. The flow has also been found to be continuous in the pipe.

4. Velocity vectors in Fig. 5 shows the direction of fluid flow. Under the influence of Kelvin body force, fluid starts to move in clockwise direction, extracting heat from the heat source and during its passage dissipating thermal energy to the surroundings through fins attached at the lower end of the closed loop, thus low temperature fluid is always available at the end of every cycle.

The numerical study conducted in this paper signifies the heat transfer capability of ferrofluid as a coolant. The system as a whole is totally passive as it does not require any external device such as pump for circulating the fluid. Moreover, there are no rotating/reciprocating parts, thus operation is noise-free and reliability of the system is excellent. Further research could be carried out to find optimal dimensions of the loop and best combination of different properties that may maximize the thermal performance of the ferrofluid based cooling system.

\section{REFERENCES}

[1] J Singh Mehta, R Kumar, H Kumar andH Garg, “Convective Heat Transfer Enhancement Using Ferrofluid: A Review,”ASME. J. Thermal Sci. Eng. Appl, Vol. 10, No. 2, pp. 020801-12, 2017. doi:10.1115/1.4037200.

[2] A.Shakibaand K.Vahedi, "Numerical Analysis of Magnetic Field Effects on Hydro-Thermal Behavior of a Magnetic Nanofluid in a Double Pipe Heat Exchanger,’J. Magn. Magn. Mater., Vol. 402, pp. 131-142, 2016.

[3] H.Aminfar, M.Mohammadpourfard and S.Ahangar Zonouzi, "Numerical Investigation of the Transient Hydrothermal Behavior of a Ferrofluid Flowing Through a Helical Duct in the Presence of Nonuniform Magnetic Field,"ASME J. Heat Transfer, Vol. 136, No. 6, pp. 061702, 2014.

[4] M.Goharkhah, M.Ashjaeeand M.Shahabadi, "Experimental Investigation on Convective Heat Transfer and Hydrodynamic Characteristics of Magnetite Nanofluid Under the Influence of an Alternating Magnetic Field,"Int. J. Therm. Sci.,Vol. 99, pp. 113-124, 2016.

[5] E.Wrobel, Fornalik-Wajs and J.S. Szmyd, "Experimental and numerical analysis of thermo-magnetic convection in a vertical 
annular enclosure,"International Journal of Heat and Fluid Flow, Vol. 31, pp. 1019-103, 2010.

[6] R. Zanella et al.,"Study of Magnetoconvection Impact on a Coil Cooling by Ferrofluid with a Spectral/Finite-Element Method," IEEE Transactions on Magnetics,Vol. 54, No. 3, pp. 1-4,March 2018, doi: 10.1109/TMAG.2017.2749539.

[7] Eskil Aursand, Magnus Aa. Gjennestad, Karl Yngve Lervåg and Halvor Lund, "A multi-phase ferrofluid flow model with equation of state for thermomagnetic pumping and heat transfer," Journal of Magnetism and Magnetic Materials,Vol. 402, pp. 8-19, 2016.doi: 10.1016/j.jmmm.2015.11.042,.
[8] Tomasz Strek, "Finite Element Analysis of Ferrofluid Cooling of Heat Generating Devices,"Proceedings of the COMSOL Conference 2008 Hannover, 2008.

[9] W. Cherief, Y. Avenas, S. Ferrouillat, A. Kedous-Lebouc, L. Jossic and M. Petit, "Parameters affecting forced convection enhancement in ferrofluid cooling systems,"Appl. Therm. Eng., Vol. 123, pp. 156166, 2017.

[10] R. E.Rosensweig, Ferrohydrodynamics, Cambridge University Press, New York, 1985.

[11] S.Odenbach, "Magnetoviscous Effects in Ferrofluids",Springer, 2002.

[12] S.Odenbach (red.), "Ferrofluids: Magnetically Controllable Fluids and Their Applications,”Springer, 2002. 